\title{
Titer regulation in arthropod-Wolbachia symbioses
}

\author{
Sergio López-Madrigal ${ }^{1}$, Elves H Duarte
}

Keywords: Wolbachia, arthropods, symbiosis, titer modulation, co-adaptation, evolution

\begin{abstract}
Symbiosis between intracellular bacteria (endosymbionts) and animals are widespread. The alphaproteobacterium Wolbachia pipientis is known to maintain a variety of symbiotic associations, ranging from mutualism to parasitism, with a wide range of invertebrates. Wolbachia infection might deeply affect host fitness (e.g. reproductive manipulation, antiviral protection), which is thought to explain its high prevalence in nature. Bacterial loads significantly influence both the infection dynamics and the extent of bacteria-induced host phenotypes. Hence, fine regulation of bacterial titers is considered as a milestone in host-endosymbiont interplay. Here we review both environmental and biological factors modulating Wolbachia titers in arthropods.
\end{abstract}

\section{INTRODUCTION}

Symbiotic associations between bacteria and animals are widespread, significantly affecting animals' fitness and hence critically influencing their evolutionary dynamics (Douglas 2014). Most of these bacteria colonize external or internal surfaces of the host's body. Moreover, some of them deeply integrate into animals' biology by stably colonizing intracellular niches (Fisher et al. 2017). Symbiosis with intracellular bacteria (endosymbionts) is particularly common in invertebrates, whose huge diversification partially relies on bacteria-derived fitness advantages (Mandel and Dunn, 2016; Sudakaran et al. 2017; Pollock et al. 2018). Endosymbionts are either essential or facultative. Essential endosymbionts (also known as primary, P-endosymbionts) are strictly intracellular bacteria required for proper host development and reproduction. They are fixed in the host population and get vertically transmitted (i.e. from mothers to offspring). In contrast, facultative symbionts (also known as secondary, S-symbionts) typically colonize a broader range of tissues both intra- and extra-cellularly. S-symbionts might behave as parasites and/or supply their host a variety of non-essential fitness advantages. They infect a subset of individuals in a host population and can be transmitted vertically and horizontally. Endosymbionts should be abundant enough to play their symbiotic role, limit their infection cost and ensure at least, their vertical inheritance between host generations (Vigneron et al. 2014; Simonet et al. 2016; Campbell et al. 2018).

The alphaproteobacterium Wolbachia pipientis (Proteobacteria: Anaplasmataceae) is among the most widespread bacterial endosymbionts in nature, infecting filarial nematodes and arthropods (Gerth et al. 2014). Wolbachia evolutionary success relies on its vertical transfer between host generations. Accordingly, it colonizes the germ line early during host development (Serbus et al. 2008). Horizontal transfer of Wolbachia also

\footnotetext{
${ }^{1}$ Corresponding author:

Sergio López-Madrigal. Instituto Gulbenkian de Ciência. Rua da Quinta Grande, 6. 2780-156 Oeiras,

Portugal; Tel: +351-214464516; Fax: +351-214407970; Email: slopez@igc.gulbenkian.pt
} 
occurs, as suggested by discrepancies between bacteria and host phylogenetic topologies (Ahmed et al. 2016). It might be favored by common cellular mechanisms allowing for host cell infection (White et al. 2017a) and seems to take place in a variety of ecological scenarios (Huigens et al. 2004; Le Clec'h et al. 2013; Ahmed et al. 2015; Brown and Lloyd 2015).

Wolbachia is generally seen as an opportunistic reproductive parasite which is able to favor infection persistence and spread by manipulating its host's reproduction in several ways (i.e. cytoplasmic incompatibility, parthenogenesis, male-killing, feminization of genetic males) (Werren et al. 2008). Nevertheless, Wolbachia can play a variety of roles in symbiotic associations. For instance, it supplies non-essential fitness advantages to many invertebrate species, including behavioral modifications and resistance against bacteria, viruses and Plasmodium parasite infection (de Crespigny et al. 2006; Hedges et al. 2008; Teixeira et al. 2008; Hughes et al. 2011; Furihata et al. 2015; Rohrscheib et al. 2015; Braquart-Varnier et al. 2015a). Moreover, Wolbachia behaves as an obligate nutritional mutualist in some insect and nematode lineages (Hellemans et al. 2019; Nikoh et al. 2014; Balvín et al. 2018; Shaikevich and Ganushkina, 2018). In this context, Wolbachia genome size varies according to its host dependency, ranging from $0.96 \mathrm{Mb}$ ( $w$ Ov and wOo strains, Darby et al. 2012; Desjardins et al. 2013) to $1.80 \mathrm{Mb}$ ( $w$ Fol strain, Faddeeva-Vakhrusheva et al. 2017). Thus, with very few exceptions (Kampfraath et al. 2019), the genomes of obligate mutualistic strains are generally smaller than those of Ssymbionts and/or reproductive manipulators.

Both Wolbachia-induced host phenotypes and transmission potential are highly influenced by Wolbachia loads. Given its broad phylogenetic distribution (Hilgenboecker et al. 2008), Wolbachia-involving symbioses represent an excellent target for the study of mechanisms driving the evolution of bacteria-animal symbioses. Here we review the current knowledge on factors and mechanisms shaping Wolbachia titers in arthropods and its relevance for the stability of symbiotic associations.

\section{WOLBACHIA LOADS MATTER}

Titers critically influence the stability of Wolbachia infection and the extent of Wolbachia-induced host phenotypes (Boyle et al. 1993, Dedeine et al. 2001, Veneti et al. 2004, Unckless et al. 2009, Osborne et al. 2012, Pan et al. 2018; Baião et al. 2019). Therefore, titer is a key parameter for the understanding of Wolbachia-host evolutionary dynamics and for the efficient implementation of Wolbachia as a control tool against insect pests and vector-borne diseases (Hoffmann et al. 2015).

\section{Wolbachia transmission}

Bacterial titers should be high enough to allow for the infection of tissues mediating Wolbachia vertical transfer between host generations. Consistently, Wolbachia is highly abundant in ovaries and early developmental stages of Drosophila (Diptera: Drosophilidae) oocytes, promoting colonization of embryo's germ line via mass action and strategic subcellular localization (Veneti et al. 2004). Low titers might impede Wolbachia vertical transmission yielding cured animals or, in the case of co-infection, leading to the stochastic loss of particular Wolbachia strains. For instance, the parasitoid wasp Nasonia vitripennis (Hymenoptera: Pteromalidae) infected with multiple 
Wolbachia strains yields both mono-infected and aposymbiotic progeny after prolonged larval diapause (Perrot-Minnot et al. 1996), which has been linked to titers decline in Aedes albopictus (Diptera: Culicidae) (Ruang-areerate et al. 2004). On the other hand, Wolbachia horizontal transfer might also be critically influenced by titers, tissue tropism and host biology, which would explain differences in infection acquisition/loss rates among different taxonomic groups (Bailly-Bechet et al. 2017). High bacterial density in highly abundant/accessible tissues is expected to favor horizontal spread upon certain ecological scenarios (e.g. cannibalism, predation) (Le Clec'h et al. 2013). In support of this hypothesis, a significant rise of bacterial titers in the hemolymph was detected after several forced Wolbachia horizontal passage events in Armadillidium vulgare (Isopoda: Armadillidiidae) (Le Clec'h et al. 2017). Along the same lines, Wolbachia infection enhances the host-searching ability of the parasitoid wasp Asobara japonica (Hymenoptera: Braconidae), so that Drosophila melanogaster (Diptera: Drosophilidae) larvae are more actively parasitized by infected female wasps (Furihata et al. 2015). Taking into account evidence suggesting that parasitoids can mediate Wolbachia horizontal transfer between unrelated hosts (Ahmed et al. 2015), Wolbachia impact on the ovipositioning behaviour of $A$. japonica might promote infection spread. Whether such a phenotype is linked or not to Wolbachia density remains unclear though.

Besides ensuring infection spread, high bacterial titers might favor infection persistence by allowing Wolbachia to overcome stressing environmental conditions or host's defenses (Braquart-Varnier et al. 2008; Le Clec'h et al. 2013; Pan et al. 2018).

\section{Wolbachia-induced host phenotypes}

Reproductive manipulation. Wolbachia is primarily known as a reproductive manipulator, since infection can affect its host fertility, mating behavior and/or the sexratio of host progeny (Dedeine et al. 2001; Kremer et al. 2009). Cytoplasmic incompatibility (CI) is the most widespread Wolbachia-induced phenotype causing reproductive manipulation. It induces embryonic lethality in crosses involving Wolbachia-infected males and uninfected females, thus favoring the spread of Wolbachia infection by enhancing the fitness of Wolbachia-infected females (Boyle et al. 1993; Veneti et al. 2004; Hughes and Rasgon, 2014). The strength of CI has been linked to Wolbachia titers either directly or indirectly (i.e. CI levels correlate with environmental or biological factors previously associated with Wolbachia titers variation) (Clancy and Hoffmann, 1998; Noda et al. 2001; Calvitti et al. 2015). Nevertheless, CI strength/Wolbachia density association is not universal, varying between different species or even at the population level within the same host species (Mouton et al. 2006; Duron et al. 2006; Richardson et al. 2019; Yang et al. 2019). Moreover, multiple Wolbachiainduced phenotypes might co-occur at the same time, so that infection effects on Wolbachia population dynamics are complex. For example, Wolbachia influence on the mating behavior of male flies seems to buffer the effect of CI. In this context, males of D. melanogaster and Drosophila simulans (Diptera: Drosophilidae) mate at a higher rate when infected with Wolbachia (de Crespigny et al. 2006; Awrahman et al. 2014). Higher male promiscuity restores the reproductive compatibility with uninfected females, potentially undermining the CI-based transmission advantage of Wolbachia. Lower infection frequencies yielded by this effect might favor infection losses in the host population, regardless of Wolbachia titers within individual hosts. 
Many other Wolbachia-induced phenotypes leading to reproductive manipulation have been reported (Werren et al. 2008). Phenotype strength/Wolbachia density association is mostly unclear though.

Pathogen protection. Wolbachia is known to protect their hosts from pathogen infection. Molecular mechanisms driving protection likely involve both Wolbachia and host factors (Ford et al. 2019). They remain unclear though, possibly varying between different symbiotic models. Wolbachia-mediated activation of the immune system (Pan et al. 2018) or Wolbachia/pathogen competition for space and/or resources (Caragata et al. 2013; Chrostek et al. 2014; Caragata et al. 2014; Rossi et al. 2015; Lindsey et al. 2018), both tightly related to bacterial titers, have been considered as suitable mechanisms. Consistently, several studies have already linked Wolbachia loads to the extent of Wolbachia-mediated protective phenotypes, so that highly protective strains use to occur at higher titers (Osborne et al. 2012; Chrostek et al. 2013; Chrostek et al. 2014; Martinez et al. 2014; Martinez et al. 2017).

Infection cost. Wolbachia infection might significantly affect many life-history traits of the host (e.g. fecundity, lifespan). Association between infection cost and Wolbachia loads (i.e. the higher the titers the stronger the Wolbachia-induced phenotype) have been suggested (Suh et al. 2009; Henry and Newton, 2018; Baião et al. 2019), although it needs to be further analyzed in most of the reported cases (Hamm et al. 2014; Suh et al. 2017). For instance, lifespan shortening of infected hosts was noted early in the research field and has been shown in several arthropod lineages (e.g. Aedes, Armadillidium, Drosophila) (Min and Benzer 1997; Suh et al. 2009; Chrostek et al. 2013; Chrostek et al. 2014; Le Clec'h et al. 2017). Nevertheless, lifespan shortening/Wolbachia titers association is not universal, as already seen for other Wolbachia-induced host phenotypes (Braquart-Varnier et al. 2008).

\section{FACTORS AND MECHANISMS DRIVING WOLBACHIA LOADS}

\section{Environmental factors}

Temperature. Environmental temperature displays a great impact over Wolbachia titers and titer-dependent host phenotypes (e.g. shortening of host lifespan) (Figure 1). In this context, temperatures over $30^{\circ} \mathrm{C}$ lower Wolbachia titers or even remove the infection (Opijnen and Breeuwer 1999) while temperatures below $20^{\circ} \mathrm{C}$ deeply compromise its proliferation rate. In between, titers seem to positively correlate with temperature as suggested by infection cost variations in D. melanogaster (Reynolds et al. 2003). Strikingly, Wolbachia-associated variations in host thermal preference have been reported (Truitt et al. 2018) (see Wolbachia-induced host phenotypes section for further examples of Wolbachia-induced host phenotypes). It is important to keep in mind that temperature effects on Wolbachia titers can be indirect, affecting other members of the host microbiota (see Host microbiota section) or the host biology itself. For instance, increased temperature affects Wolbachia proliferation in the brain of adult $D$. melanogaster but not in late larvae (Strunov et al. 2013). Sex-dependent, developmental stage-dependent or even cross-generational effects of temperature on Wolbachia titers have been reported so far (Clancy and Hoffmann, 1998; Foo et al. 2019). 
Host diet. As for any other endosymbiont, the host physiology defines Wolbachia chemical environment. In this context, diet composition is known to influence both host physiology and Wolbachia titers (Figure 1). For instance, several natural sugars (i.e. sucrose, galactose, lactose, maltose and trehalose) increase Wolbachia titers in developing oocytes while dietary yeast reduces it (Serbus et al. 2015; Camacho et al. 2017). Interestingly, dietary yeast effect is tissue-dependent, leading to redistribution of Wolbachia titers within the host body (Serbus et al. 2015; Christensen et al. 2019). Importantly, concerns have been recently riced on relative quantification of Wolbachia titers in this field, since diet-derived effects on the host might lead to distorted conclusions (Ponton et al. 2015; Christensen et al. 2019). Finally, antibiotics intake can significantly affect Wolbachia titers within very few host generations. Susceptibility may depend on the Wolbachia strain, the antibiotic type, concentration and ingestion regime (Fenollar et al. 2003; Liu et al. 2014; Fallon et al. 2018).

Host microbiota. Wolbachia is not alone within its host. Multiple Wolbachia strains may co-infect a single individual (Ijichi et al. 2002; Hiroki et al. 2004; Mouton et al. 2006; Valette et al. 2013). Moreover, Wolbachia usually co-exists with other endosymbiotic bacteria or members of the gut microbiota (Gómez-Valero et al. 2004; Dittmer et al. 2016). Since Wolbachia occupies a within-host niche and feeds on resources therein, coinfection of multiple bacterial lineages might translate into competition for space and nutrients (Figure 1) (Caragata et al. 2014; Geoghegan et al. 2017; Jiménez et al. 2019). For example, Spiroplasma sp. strain NSRO (Firmicutes: Spiroplasmataceae) negatively affects Wolbachia titers in D. melanogaster (Goto et al. 2006). Moreover, competitive exclusion of Wolbachia by Asaia sp. (Proteobacteria: Acetobacteraceae) occurs in Anopheles (Diptera: Culicidae), so that Wolbachia maternal transmission is inhibited (Hughes et al. 2014). Asaia is also a vertically transmitted endosymbiont colonizing mosquito germ line (Favia et al. 2007). Therefore, Asaia and Wolbachia may compete for the same niche in the female gonads (Rossi et al. 2015).

\section{Host-based mechanisms}

Distortion of bacterial titers in hybrid hosts strongly suggest that infected animals are actively controlling Wolbachia population dynamics. For instance, a dramatic increase of Wolbachia titers is observed in hybrids between members of the tsetse fly Glossina morsitans (Diptera: Glossinidae) group compared to non-hybrid hosts (Schneider et al. 2013). Along the same lines, transinfection-based approaches (i.e. Wolbachia strains transfer into an heterologous host) have suggested that host might play a key role in titer regulation (Boyle et al. 1993; Hughes and Rasgon, 2014). Consistently, transinfection has shown the impact of exogenous bacterial strains on the recipient host to be broader than that of endogenous strains (Christensen et al. 2016). For instance, major perturbation of symbiosis was observed when infecting the naïve wasp Nasonia giraulti (Hymenoptera: Pteromalidae) with a Wolbachia strain from its close relative $N$. vitripennis (Chafee et al. 2011; Funkhouser-Jones et al. 2018). Authors showed that most of Wolbachia density suppression capabilities can be mapped to two regions of the Nasonia genome and identified the Wolbachia density suppressor $(w d s)$ gene, which dominantly hinders bacterial transmission from mothers to offspring (Funkhouser-Jones et al. 2018) (Table 1). Similarly, the mosquito Aedes aegypti (Diptera: Culicidae) metalloprotease $\mathrm{m} 41 \mathrm{ftsh}$ was shown to foster Wolbachia strain wMelPop-CLA proliferation via Wolbachia- 
induced expression of the mosquito microRNA (miRNA) aae-miR-2940 (Hussain et al. 2011) (Table 1). Overexpression of miRNA aae-miR-12 in infected A. aegypti seems also relevant for replication/maintenance within host cells (Osei-Amo et al. 2012). Along the same lines, introgression analysis between two strains of Culex quinquefasciatus (Diptera: Culicidae) showing consistent differences in Wolbachia density led to the identification of two major-effect quantitative-trait loci (QTL) controlling bacterial loads in nongonadal tissues (Emerson and Glaser, 2017) (Table 1).

Developmental dynamics. Both host physiology and tissue repertoire vary significantly during animal development. Consistently, Wolbachia loads and Wolbachia-induced host phenotypes are tightly modulated by host ontogeny (Figure 1) (Ijichi et al. 2002; Reynolds et al. 2003; Ruang-areerate et al. 2004; Unckless et al. 2009; Gutzwiller et al. 2015; Voronin et al. 2012; Fisher et al. 2018). For instance, the fly axis-determinant gene Gurken ( $g r k$ ), displays a cumulative, dosage-sensitive effect on Wolbachia titers at $D$. melanogaster oogenesis (Serbus et al. 2011) (Table 1). Likewise, Wolbachia is known to accumulate in polar cells but not in lateral follicle cells during oogenesis (Kamath et al. 2018). Along the same lines, host cell autophagy has been shown to be a key negative regulator of Wolbachia titers along host ontogeny (Voronin et al. 2012). Consistently, developmental stage-dependent shifts in Wolbachia gene expression are observed along D. melanogaster development, even when considering total within-host population (Gutzwiller et al. 2015; Rice et al. 2017). Affected loci include ankyrin repeat domain (ANK)-containing genes as well as genes with predicted membrane or secretion system function, suggesting that Wolbachia-host cross-talk is influenced by host development.

Tissue tropism. Immune responses play an important role in the host-based modulation of bacteria tissue tropism (Masson et al. 2016). Both in vivo and in vitro studies have shown Wolbachia-mediated activation of immune genes and processes (Xi et al. 2008; Voronin et al. 2012; Grobler et al. 2018; Baião et al. 2019). Boosting of host immune system may also favor Wolbachia infection (Rio et al. 2006; Pan et al. 2018). For example, Wolbachia is known to activate the basal immune response of $A$. aegypti via the immune deficiency (IMD)- and Toll-pathway. Curiously, silencing of these immune pathways leads to the reduction of Wolbachia titers (Pan et al.2018). Immune activation might help Wolbachia, which apparently lacks specific targets for the corresponding antimicrobial peptides, to exclude sensitive microbial competitors. Moreover, it might also actively promote Wolbachia proliferation via parallel production of antioxidants (Pan et al. 2012; Brennan et al. 2012; Zug and Hammerstain, 2015). In spite of this, Wolbachia infection/transinfection does not necessarily lead to host immune activation. In this context, transfer of strain $w \mathrm{Au}$, native to $D$. simulans, into $D$. melanogaster led to stronger Wolbachia-induced phenotypes in comparison with strain $w \mathrm{Mel}$ performance with no apparent activation of host immune system (Chrostek et al. 2014).

Tissue tropism affects bacterial sensitivity to environmental stress and influences the extent of titer-dependent host phenotypes (Osborne et al. 2012; Strunov and Kiseleva, 2016; Shan et al. 2017). High titers and broad tissue tropism normally co-occur with stronger Wolbachia-induced host phenotypes (Mouton et al. 2004; Duron et al. 2006; Osborne et al. 2012; Baton et al. 2013). Moreover, tissue tropism is central to nutritional symbioses. Mutualist and potentially mutualist Wolbachia strains $w \mathrm{Cle}$ and $w \mathrm{Ctub}$, from Cimex lectularius (Hemiptera: Cimicidae) and Cavitermes tuberosus (Isoptera: 
Termitidae) respectively, mostly reside in an organ-like structure called bacteriome rather than maintain a more ubiquitous tissue distribution (Hosokawa et al. 2010; Hellemans et al. 2019). Finally, infection tropism involving both germ and somatic cell lines influences Wolbachia spread within and between individual hosts (Frydman et al. 2006; BraquartVarnier et al. 2015b).

Host tissues colonized by Wolbachia define the nature of the molecular cross-talk between host and endosymbiont, including gene networks allowing for colonization, maintenance and proliferation of Wolbachia. In this context, vertical inheritanceassociated bottlenecking of Wolbachia infection seems reinforced in D. melanogaster heterozygous for cytoskeletal mutations in profilin or villin (i.e. chic $^{221}$, chic $^{1320}$, qua ${ }^{6-396}$ ) (Table 1). Titers decrease co-occurs with stochastic loss of Wolbachia infection, which suggests that Wolbachia utilizes the host actin cytoskeleton for persistence within and transmission between fly generations (Newton and Sheehan, 2015; Newton et al. 2015). Moreover, genome-wide RNAi screenings on Wolbachia-infected D. melanogaster cell line JW18 have recently identified a wide set of genes as positive- or negative-regulators of Wolbachia titers, respectively (White et al. 2017b; Grobler et al. 2018). Negative regulators include many components of the ribosome, the proteasome and the regulatory proteins network. On the other hand, most of positive regulators were genes involved in metabolism and metabolite transportation, including loci involved in lipid metabolism and mitochondrial function. Strikingly, positive covariation of mitochondria and Wolbachia titers has been shown in D. melanogaster (Henry and Newton, 2018). Moreover, some genes involved in ubiquitin and proteolysis pathways were shown to behave as positive regulators of Wolbachia titers. Further functional studies have suggested that Wolbachia relies on host proteolysis via ubiquitination and Endoplasmic Reticulum (ER)-associated protein degradation pathway (ERAD) for essential amino acids provisioning (White et al. 2017b). Consistently, tightly coupled Wolbachia/ER dynamics during cell colonization has been recently shown, although gene expression and immunostaining studies did not reveal ER stress or enhanced ERAD-driven proteolysis (Fattouh et al. 2019). This apparent discrepancy is hard to interpret though, since different experimental approaches and/or cell lines were used. Finally, genome-wide RNAi screening of Wolbachia-infected JW18 cells also showed up to 166 genes to affect Wolbachia and host cell proliferation in the same way, either positive or negative, which might contribute to the in vivo coordination of bacteria/host dynamics (Grobler et al. 2018).

\section{Endosymbiont-based mechanisms}

Substantial variations have been observed in Wolbachia titers within the same host species in nature (Unckless et al. 2009). Variability remains even when rearing infected animals under controlled environmental conditions or when considering different Wolbachia strains within the same host individual, which suggests bacteria-derived factors to influence titer dynamics (Ijichi et al. 2002; Veneti et al. 2004; Osborne et al. 2012; Dittmer et al. 2014). Differences in titers do not necessarily reflect differences in proliferation rates. On the contrary, different tissue tropisms might contribute via competition with co-infective microbes or susceptibility to environmental and/or hostderived factors (Figure 1) (Ijichi et al. 2002; Veneti et al. 2004; Osborne et al. 2012; Dittmer et al. 2014). Strikingly, closely related Wolbachia strains might show similar 
titers and/or induce similar titer-dependent host phenotypes, which suggests titer regulation by shared molecular mechanisms (Veneti et al. 2004; Osborne et al. 2012; Chrostek et al. 2013). As for the study of host-based mechanisms, transinfection of different Wolbachia strains into the same host species has yielded great insight into bacteria-derived factors modulating infection density. In this context, host/Wolbachia shuffling for a variety of Drosophila species showed titers and titer-dependent phenotype (i.e. antiviral protection) to be mostly influenced by Wolbachia genotype (Martinez et al. 2014; Martinez et al. 2017). Furthermore, Wolbachia-based control of titers and titerdependent antiviral protection differs even within different variants of $w$ Mel strain (Chrostek et al. 2013). Genomic analysis of the highly proliferative Wolbachia strain $w$ MelPop (Min and Benzer 1997; Strunov and Kiseleva 2016) showed the amplification of an eight gene-containing region named Octomom to determine both Wolbachia titers and virulence (i.e. Wolbachia-induced shortening of D. melanogaster lifespan) (Chrostek et al. 2013; Chrostek and Teixeira, 2015) (Table 1). Octomom expansion, likely enhanced by selection at the intra-host level (Chrostek and Teixeira, 2018), is the first described link between Wolbachia genotype and phenotype. More recently, overexpression of the Wolbachia actin-localizing effector 1 (WalE1)-encoding gene has shown to increase bacterial titers in D. melanogaster (Sheehan et al. 2016). WalE1 is a candidate Type IV effector likely mediating Wolbachia interaction with host cell actin cytoskeleton. This gene expression is upregulated in late larval development of flies, peaking at early pupation when adult organogenesis takes place. It might affect Wolbachia titers either through bacterial proliferation or bacterial transmission (Sheehan et al. 2016) (Table 1).

\section{CONCLUSION}

Wolbachia is a maternally-inherited bacterium that stably infects a broad range of animal lineages, being therefore an excellent model for the study of mechanisms driving the evolution of bacteria-animal symbioses. Wolbachia needs to guarantee infection persistence between host generations, either supplying the host with any fitness advantage and/or actively enhancing infection spread via reproductive manipulation. Both the extent of infection-induced host phenotypes and its spread potential, either vertical or horizontal, might be highly influenced by Wolbachia titers. Thus, fine regulation of Wolbachia loads is a key milestone in the evolution of Wolbachia-host symbioses.

Titers may vary when considering different Wolbachia strains, host species or host populations. Significant variations are frequent even within the same host population, at the individual level, for the same Wolbachia strain. Altogether, it suggests that Wolbachia density dynamics is driven by a wide spectrum of factors, either extrinsic or intrinsic, and their potential interplay. Thus, model- or context-dependent traits rather than universal mechanisms operating all over Wolbachia/arthropod symbioses have been reported.

Extrinsic factors represent environmental conditions in a broad sense, thus considering physical, chemical and biological parameters. In contrast, intrinsic factors include Wolbachia- and host-based mechanisms shaping bacteria density and distribution. The impact of extrinsic factors on Wolbachia titers has been extensively studied. Available data should be carefully interpreted, since direct effects on Wolbachia are hard to prove. In contrast, very little is still known on the nature and role of intrinsic factors, which likely involve a great variety of genes. So far several loci, either prokaryotic or eukaryotic, are 
known to play a role in the in vivo modulation of Wolbachia titer. Furthermore, plenty of candidate loci awaiting for experimental validation have been shown by a variety of highthroughput screenings.

Genes driving the molecular cross-talk between Wolbachia and its host are expected to evolve under simultaneous selective pressures both at individual- and population-level. Evolutionary dynamics of these loci may be highly complex, since (a) selective pressures within- and between-host individuals might oppose each other (i.e. favoring Wolbachiadriven infection spread or host-driven infection control, respectively) and (b) the impact of Wolbachia on host fitness may greatly depend on the interplay of extrinsic and intrinsic factors. Regardless of the ecological scenario, a fine trade-off between Wolbachia and host fitness is required for the symbiosis to be stable over time.

\section{CONFLICTS OF INTEREST}

Authors declare no conflict of interest

\section{FUNDING}

This work was supported by Instituto Gulbenkian de Ciência and the European Research Council Executive Agency (773260-WOLBACKIAN). EHD is a recipient of a $\mathrm{PhD}$ fellowship from Fundação para a Ciência e Tecnologia (SFRH/BD/113757/2015) under the Graduate Program Science for the Development (PGCD).

\section{ACKNOWLEDGMENTS}

Authors would like to thank Luís Teixeira and Ana Carvalho for insightful comments on the manuscript.

\section{REFERENCES}

Ahmed MZ, Breinholt JW, Kawahara AY. Evidence for common horizontal transmission of Wolbachia among butterflies and moths. BMC Evol Biol 2016;16:118.

Ahmed MZ, Li S-J, Xue X et al. The intracellular bacterium Wolbachia uses parasitoid wasps as phoretic vectors for efficient horizontal transmission. PLOS Pathog 2015;11:e1004672.

Awrahman ZA, Champion de Crespigny F, Wedell N. The impact of Wolbachia, male age and mating history on cytoplasmic incompatibility and sperm transfer in Drosophila simulans. J Evol Biol 2014;27:1-10.

Baião GC, Schneider DI, Miller WJ et al. The effect of Wolbachia on gene expression in Drosophila paulistorum and its implications for symbiont-induced host speciation. BMC Genomics 2019;20:465.

Bailly-Bechet M, Martins-Simões P, Szöllosi GJ et al. How long does Wolbachia remain on board? Mol Biol Evol 2017;34:1183-93.

Balvín O, Roth S, Talbot B et al. Co-speciation in bedbug Wolbachia parallel the pattern in nematode hosts. Sci Rep 2018;8:8797. 
Baton LA, Pacidônio EC, Gonçalves DS et al. wFlu: Characterization and evaluation of a native Wolbachia from the mosquito Aedes fluviatilis as a potential vector control agent. PLoS One 2013;8:e59619.

Boyle L, O'Neill S, Robertson H et al. Interspecific and intraspecific horizontal transfer of Wolbachia in Drosophila. Science 1993;260:1796-9.

Braquart-Varnier C, Altinli M, Pigeault R et al. The mutualistic side of Wolbachiaisopod interactions: Wolbachia mediated protection against pathogenic intracellular bacteria. Front Microbiol 2015;6:1388.

Braquart-Varnier $\mathrm{C}$, Lachat $\mathrm{M}$, Herbinière $\mathrm{J}$ et al. Wolbachia mediate variation of host immunocompetence. PLoS One 2008;3:e3286.

Braquart-Varnier C, Raimond M, Mappa G et al. The hematopoietic organ: a cornerstone for Wolbachia propagation between and within hosts. Front Microbiol 2015b;6:1424.

Brennan LJ, Haukedal JA, Earle JC et al. Disruption of redox homeostasis leads to oxidative DNA damage in spermatocytes of Wolbachia-infected Drosophila simulans. Insect Mol Biol 2012;21:510-20.

Brown AN, Lloyd VK. Evidence for horizontal transfer of Wolbachia by a Drosophila mite. Exp Appl Acarol 2015;66:301-11.

Calvitti M, Marini F, Desiderio A et al. Wolbachia density and cytoplasmic incompatibility in Aedes albopictus: concerns with using artificial Wolbachia infection as a vector suppression tool. PLoS One 2015;10:e121813.

Camacho M, Oliva M, Serbus LR. Dietary saccharides and sweet tastants have differential effects on colonization of Drosophila oocytes by Wolbachia endosymbionts. Biol Open 2017;6:1074-83.

Campbell MA, Łukasik P, Meyer MC et al. Changes in endosymbiont complexity drive host-level compensatory adaptations in cicadas. MBio 2018;9:e2104.

Caragata EP, Rancès E, Hedges LM et al. Dietary cholesterol modulates pathogen blocking by Wolbachia. PLoS Pathog 2013;9:e1003459.

Caragata EP, Rancès E, O'Neill SL et al. Competition for amino acids between Wolbachia and the mosquito host, Aedes aegypti. Microb Ecol 2014;67:205-18.

Chafee ME, Zecher CN, Gourley ML et al. Decoupling of host-symbiont-phage coadaptations following transfer between insect species. Genetics 2011;187:203-15.

Christensen S, Camacho M, Sharmin Z et al. Quantitative methods for assessing local and bodywide contributions to Wolbachia titer in maternal germline cells of Drosophila. BMC Microbiol 2019;19:206. 
Christensen S, Pérez Dulzaides R, Hedrick VE et al. Wolbachia endosymbionts modify Drosophila ovary protein levels in a context-dependent manner. Appl Environ Microbiol 2016;82:5354-63.

Chrostek E, Marialva MSP, Esteves SS et al. Wolbachia variants induce differential protection to viruses in Drosophila melanogaster: a phenotypic and phylogenomic analysis. PLoS Genet 2013;9:e1003896.

Chrostek E, Marialva MSP, Yamada R et al. High Anti-viral protection without immune upregulation after interspecies Wolbachia transfer. PLoS One 2014;9:e99025.

Chrostek E, Teixeira L. Mutualism breakdown by amplification of Wolbachia genes. PLOS Biol 2015;13:e1002065.

Chrostek E, Teixeira L. Within host selection for faster replicating bacterial symbionts. PLoS One 2018;13:e0191530.

Clancy DJ, Hoffmann AA. Environmental effects on cytoplasmic incompatibility and bacterial load in Wolbachia-infected Drosophila simulans. Entomol Exp Appl 1998;86:13-24.

Darby AC, Armstrong SD, Bah GS et al. Analysis of gene expression from the Wolbachia genome of a filarial nematode supports both metabolic and defensive roles within the symbiosis. Genome Res 2012;22:2467-77.

de Crespigny F, Pitt T, Wedell N. Increased male mating rate in Drosophila is associated with Wolbachia infection. J Evol Biol 2006;19:1964-72.

Dedeine F, Vavre F, Fleury F et al. Removing symbiotic Wolbachia bacteria specifically inhibits oogenesis in a parasitic wasp. Proc Natl Acad Sci U S A 2001;98:6247-52.

Desjardins CA, Cerqueira GC, Goldberg JM et al. Genomics of Loa loa, a Wolbachiafree filarial parasite of humans. Nat Genet 2013;45:495-500.

Dittmer J, Beltran-Bech S, Lesobre J et al. Host tissues as microhabitats for Wolbachia and quantitative insights into the bacterial community in terrestrial isopods. Mol Ecol 2014;23:2619-35.

Dittmer J, Lesobre J, Moumen B et al. Host origin and tissue microhabitat shaping the microbiota of the terrestrial isopod Armadillidium vulgare. FEMS Microbiol Ecol 2016;92:fiw063.

Douglas AE. Symbiosis as a general principle in eukaryotic evolution. Cold Spring Harb Perspect Biol 2014;6:a016113.

Duron O, Labbé P, Berticat C et al. High Wolbachia density correlates with cost of infection for insecticide resistant Culex pipiens mosquitoes. Evolution 2006;60:303-14. 
Emerson KJ, Glaser RL. Cytonuclear epistasis controls the density of symbiont Wolbachia pipientis in nongonadal tissues of mosquito Culex quinquefasciatus. G3 (Bethesda) 2017;7:2627-35.

Faddeeva-Vakhrusheva A, Kraaijeveld K, Derks MFL et al. Coping with living in the soil: the genome of the parthenogenetic springtail Folsomia candida. BMC Genomics 2017;18:493.

Fallon AM. Strain-specific response to ampicillin in Wolbachia-infected mosquito cell lines. Vitr Cell Dev Biol Anim 2018;54:580-8.

Fattouh N, Cazevieille C, Landmann F. Wolbachia endosymbionts subvert the endoplasmic reticulum to acquire host membranes without triggering ER stress. PLoS Negl Trop Dis 2019;13:e007218.

Favia G, Ricci I, Damiani C et al. Bacteria of the genus Asaia stably associate with Anopheles stephensi, an Asian malarial mosquito vector. Proc Natl Acad Sci 2007;104:9047-51.

Fenollar F, Maurin M, Raoult D. Wolbachia pipientis growth kinetics and susceptibilities to 13 antibiotics determined by immunofluorescence staining and real-time PCR. Antimicrob Agents Chemother 2003;47:1665-71.

Fisher ML, Watson DW, Osborne JA et al. Growth kinetics of endosymbiont Wolbachia in the common bed bug, Cimex lectularius. Sci Rep 2018;8:11444.

Fisher RM, Henry LM, Cornwallis CK et al. The evolution of host-symbiont dependence. Nat Commun 2017;8:15973.

Foo IJ-H, Hoffmann AA, Ross PA et al. Cross-generational effects of heat stress on fitness and Wolbachia density in Aedes aegypti mosquitoes. Trop Med Infect Dis 2019;4: E13.

Ford SA, Allen SL, Ohm JR et al. Selection on Aedes aegypti alters Wolbachia-mediated dengue virus blocking and fitness. Nat Microbiol 2019;4:1832-9.

Frydman HM, Li JM, Robson DN et al. Somatic stem cell niche tropism in Wolbachia. Nature 2006;441:509-12.

Funkhouser-Jones LJ, van Opstal EJ, Sharma A et al. The maternal effect gene Wds controls Wolbachia titer in Nasonia. Curr Biol 2018;28:1692-1702.e6.

Furihata S, Hirata M, Matsumoto H et al. Bacteria endosymbiont, Wolbachia, promotes parasitism of parasitoid wasp Asobara japonica. PLoS One 2015;10:e0140914.

Geoghegan V, Stainton K, Rainey SM et al. Perturbed cholesterol and vesicular trafficking associated with dengue blocking in Wolbachia-infected Aedes aegypti cells. Nat Commun 2017;8:526.

Gerth M, Gansauge M-T, Weigert A et al. Phylogenomic analyses uncover origin and spread of the Wolbachia pandemic. Nat Commun 2014;5:5117. 
Gómez-Valero L, Soriano-Navarro M, Perez-Brocal V et al. Coexistence of Wolbachia with Buchnera aphidicola and a secondary symbiont in the aphid Cinara cedri. $J$ Bacteriol 2004;186:6626-33.

Goto S, Anbutsu H, Fukatsu T. Asymmetrical interactions between Wolbachia and Spiroplasma endosymbionts coexisting in the same insect host. Appl Environ Microbiol 2006;72:4805-10.

Grobler Y, Yun CY, Kahler DJ et al. Whole genome screen reveals a novel relationship between Wolbachia levels and Drosophila host translation. PLOS Pathog 2018;14:e1007445.

Gutzwiller F, Carmo CR, Miller DE et al. Dynamics of Wolbachia pipientis gene expression across the Drosophila melanogaster life cycle. G3 2015;5:2843-56.

Hedges LM, Brownlie JC, O'Neill SL et al. Wolbachia and virus protection in insects. Science 2008;322:702.

Hamm CA, Begun DJ, Vo A et al. Wolbachia do not live by reproductive manipulation alone: infection polymorphism in Drosophila suzukii and D. subpulchrella. Mol Ecol 2014;23:4871-85.

Hellemans S, Kaczmarek N, Marynowska M et al. Bacteriome-associated Wolbachia of the parthenogenetic termite Cavitermes tuberosus. FEMS Microbiol Ecol 2019;95:fiy235.

Henry LP, Newton ILG. Mitochondria and Wolbachia titers are positively correlated during maternal transmission. Mol Ecol 2018;27:2634-46.

Hilgenboecker K, Hammerstein P, Schlattmann P et al. How many species are infected with Wolbachia: a statistical analysis of current data. FEMS Microbiol Lett 2008;281:215-20.

Hiroki M, Tagami Y, Miura K et al. Multiple infection with Wolbachia inducing different reproductive manipulations in the butterfly Eurema hecabe. Proc R Soc London Ser B Biol Sci 2004;271:1751-5.

Hoffmann AA, Ross PA, Rašic G. Wolbachia strains for disease control: ecological and evolutionary considerations. Evol Appl 2015;8:751-68.

Hosokawa T, Koga R, Kikuchi Y et al. Wolbachia as a bacteriocyte-associated nutritional mutualist. Proc Natl Acad Sci 2010;107:769-74.

Hughes GL, Dodson BL, Johnson RM et al. Native microbiome impedes vertical transmission of Wolbachia in Anopheles mosquitoes. Proc Natl Acad Sci 2014;111:12498-503.

Hughes GL, Koga R, Xue P et al. Wolbachia infections are virulent and inhibit the human malaria parasite Plasmodium falciparum in Anopheles gambiae. PLoS Pathog 2011;7:e1002043. 
Hughes GL, Rasgon JL. Transinfection: a method to investigate Wolbachia-host interactions and control arthropod-borne disease. Insect Mol Biol 2014;23:141-51.

Huigens ME, de Almeida RP, Boons PA et al. Natural interspecific and intraspecific horizontal transfer of parthenogenesis-inducing Wolbachia in Trichogramma wasps. Proc Biol Sci 2004;271:509-15.

Hussain M, Frentiu FD, Moreira LA et al. Wolbachia uses host microRNAs to manipulate host gene expression and facilitate colonization of the dengue vector Aedes aegypti. Proc Natl Acad Sci U S A 2011;108:9250-5.

Ijichi N, Kondo N, Matsumoto R et al. Internal spatiotemporal population dynamics of infection with three Wolbachia strains in the adzuki bean beetle, Callosobruchus chinensis (Coleoptera: Bruchidae). Appl Environ Microbiol 2002;68:4074-80.

Jiménez NE, Gerdtzen ZP, Olivera-Nappa Á et al. A systems biology approach for studying Wolbachia metabolism reveals points of interaction with its host in the context of arboviral infection. PLoS Negl Trop Dis 2019;13:e0007678.

Kamath AD, Deehan MA, Frydman HM. Polar cell fate stimulates Wolbachia intracellular growth. Development 2018;145:dev158097.

Kampfraath AA, Klasson L, Anvar SY et al. Genome expansion of an obligate parthenogenesis-associated Wolbachia poses an exception to the symbiont reduction model. BMC Genomics 2019;20:106.

Kremer N, Charif D, Henri H et al. A new case of Wolbachia dependence in the genus Asobara: evidence for parthenogenesis induction in Asobara japonica. Heredity 2009;103:248-56.

Le Clec'h W, Chevalier FD, Genty L et al. Cannibalism and predation as paths for horizontal passage of Wolbachia between terrestrial isopods. PLoS One 2013;8:e60232.

Le Clec'h W, Dittmer J, Raimond M et al. Phenotypic shift in Wolbachia virulence towards its native host across serial horizontal passages. Proc $R$ Soc B Biol Sci 2017;284:20171076.

Lindsey ARI, Bhattacharya T, Newton ILG et al. Conflict in the intracellular lives of endosymbionts and viruses: a mechanistic look at Wolbachia-mediated pathogenblocking. Viruses 2018;10:141.

Liu H-Y, Wang Y-K, Zhi C-C et al. A novel approach to eliminate Wolbachia infections in Nasonia vitripennis revealed different antibiotic resistance between two bacterial strains. FEMS Microbiol Lett 2014;355:163-9.

Mandel MJ, Dunn AK. Impact and influence of the natural Vibrio-squid symbiosis in understanding bacterial-animal interactions. Front Microbiol 2016;7:1982.

Martinez J, Longdon B, Bauer S et al. Symbionts commonly provide broad spectrum resistance to viruses in insects: a comparative analysis of Wolbachia strains. PLoS Pathog 2014;10:e1004369. 
Martinez J, Tolosana I, Ok S et al. Symbiont strain is the main determinant of variation in Wolbachia-mediated protection against viruses across Drosophila species. Mol Ecol 2017;26:4072-84.

Masson F, Zaidman-Rémy A, Heddi A. Antimicrobial peptides and cell processes tracking endosymbiont dynamics. Philos Trans R Soc B Biol Sci 2016;371:20150298.

Min K-TT, Benzer S. Wolbachia, normally a symbiont of Drosophila, can be virulent, causing degeneration and early death. Proc Natl Acad Sci 1997;94:10792-6.

Mouton L, Dedeine F, Henri H et al. Virulence, multiple infections and regulation of symbiotic population in the Wolbachia-Asobara tabida symbiosis. Genetics 2004;168:181-9.

Mouton L, Henri H, Bouletreau M et al. Effect of temperature on Wolbachia density and impact on cytoplasmic incompatibility. Parasitology 2006;132:49-56.

Newton ILG, Savytskyy O, Sheehan KB. Wolbachia utilize host actin for efficient maternal transmission in Drosophila melanogaster. PLOS Pathog 2015;11:e1004798.

Newton IL, Sheehan KB. Passage of Wolbachia pipientis through mutant Drosophila melanogaster induces phenotypic and genomic changes. Appl Environ Microbiol 2015;81:1032-7.

Nikoh N, Hosokawa T, Moriyama M et al. Evolutionary origin of insect-Wolbachia nutritional mutualism. Proc Natl Acad Sci 2014;111:10257-62.

Noda H, Koizumi Y, Zhang Q et al. Infection density of Wolbachia and incompatibility level in two planthopper species, Laodelphax striatellus and Sogatella furcifera. Insect Biochem Mol Biol 2001;31:727-37.

Osborne SE, Iturbe-Ormaetxe I, Brownlie JC et al. Antiviral protection and the importance of Wolbachia density and tissue tropism in Drosophila simulans. Appl Environ Microbiol 2012;78:6922-9.

Osei-Amo S, Hussain M, O'Neill SL et al. Wolbachia-induced aae-miR-12 miRNA negatively regulates the expression of MCT1 and MCM6 genes in Wolbachia-infected mosquito cell line. PLoS One 2012;7:e50049.

Pan X, Pike A, Joshi D et al. The bacterium Wolbachia exploits host innate immunity to establish a symbiotic relationship with the dengue vector mosquito Aedes aegypti. ISME J 2018;12:277-88.

Pan X, Zhou G, Wu J et al. Wolbachia induces reactive oxygen species (ROS)-dependent activation of the Toll pathway to control dengue virus in the mosquito Aedes aegypti. Proc Natl Acad Sci 2012;109:E23-31.

Perrot-Minnot MJ, Guo LR, Werren JH. Single and double infections with Wolbachia in the parasitic wasp Nasonia vitripennis: effects on compatibility. Genetics 1996;143:96172. 
Pollock FJ, McMinds R, Smith $\mathrm{S}$ et al. Coral-associated bacteria demonstrate phylosymbiosis and cophylogeny. Nat Commun 2018;9:4921.

Ponton F, Wilson K, Holmes A et al. Macronutrients mediate the functional relationship between Drosophila and Wolbachia. Proc R Soc B Biol Sci 2014;282:2014202920142029.

Reynolds KT, Thomson LJ, Hoffmann AA. The effects of host age, host nuclear background and temperature on phenotypic effects of the virulent Wolbachia strain popcorn in Drosophila melanogaster. Genetics 2003;164:1027-34.

Rice DW, Sheehan KB, Newton ILG. Large-scale identification of Wolbachia pipientis effectors. Genome Biol Evol 2017;9:1925-1937.

Richardson KM, Griffin PC, Lee SF et al. A Wolbachia infection from Drosophila that causes cytoplasmic incompatibility despite low prevalence and densities in males. Heredity 2019;122:428-40.

Rio RV., Wu Y, Filardo G et al. Dynamics of multiple symbiont density regulation during host development: tsetse fly and its microbial flora. Proc R Soc B Biol Sci 2006;273:80514.

Rohrscheib CE, Bondy E, Josh $\mathrm{P}$ et al. Wolbachia influences the production of octopamine and affects Drosophila male aggression. Appl Environ Microbiol 2015;81:4573-80.

Rossi P, Ricci I, Cappelli A et al. Mutual exclusion of Asaia and Wolbachia in the reproductive organs of mosquito vectors. Parasit Vectors 2015;8:278.

Ruang-areerate T, Kittayapong P, McGraw EA et al. Wolbachia replication and host cell division in Aedes albopictus. Curr Microbiol 2004;49:10-2.

Schneider DI, Garschall KI, Parker AG et al. Global Wolbachia prevalence, titer fluctuations and their potential of causing cytoplasmic incompatibilities in tsetse flies and hybrids of Glossina morsitans subgroup species. J Invertebr Pathol 2013;112:S104-15.

Serbus LR, Casper-Lindley C, Landmann F et al. The genetics and cell biology of Wolbachia-host interactions. Annu Rev Genet 2008;42:683-707.

Serbus LR, Ferreccio A, Zhukova M et al. A feedback loop between Wolbachia and the Drosophila gurken mRNP complex influences Wolbachia titer. J Cell Sci 2011;124:4299-308.

Serbus LR, White PM, Silva JP et al. The impact of host diet on Wolbachia titer in Drosophila. PLoS Pathog 2015;11:e1004777.

Shaikevich EV., Ganushkina LA. Wolbachia bacteria and filarial nematodes: mutual benefit and the parasite's Achilles' heel. Biol Bull Rev 2018;8:509-17. 
Shan H-W, Deng W-H, Luan J-B et al. Thermal sensitivity of bacteriocytes constrains the persistence of intracellular bacteria in whitefly symbiosis under heat stress. Environ Microbiol Rep 2017;9:706-16.

Sheehan KB, Martin M, Lesser CF et al. Identification and characterization of a candidate Wolbachia pipientis type IV effector that interacts with the actin cytoskeleton. MBio 2016;7:e0622.

Simonet P, Duport G, Gaget K et al. Direct flow cytometry measurements reveal a finetuning of symbiotic cell dynamics according to the host developmental needs in aphid symbiosis. Sci Rep 2016;6:19967.

Strunov A, Kiseleva E. Drosophila melanogaster brain invasion: pathogenic Wolbachia in central nervous system of the fly. Insect Sci 2016;23:253-64.

Strunov A, Kiseleva E, Gottlieb Y. Spatial and temporal distribution of pathogenic Wolbachia strain wMelPop in Drosophila melanogaster central nervous system under different temperature conditions. J Invertebr Pathol 2013;114:22-30.

Sudakaran S, Kost C, Kaltenpoth M. Symbiont acquisition and replacement as a source of ecological innovation. Trends Microbiol 2017;25:375-90.

Suh E, Mercer DR, Dobson SL. Life-shortening Wolbachia infection reduces population growth of Aedes aegypti. Acta Trop 2017;172:232-9.

Suh E, Mercer DR, Fu Y et al. Pathogenicity of life-shortening Wolbachia in Aedes albopictus after transfer from Drosophila melanogaster. Appl Environ Microbiol 2009;75:7783-8.

Teixeira L, Ferreira Á, Ashburner M et al. The bacterial symbiont Wolbachia induces resistance to RNA viral infections in Drosophila melanogaster. PLoS Biol 2008;6:e1000002.

Truitt AM, Kapun M, Kaur R et al. Wolbachia modifies thermal preference in Drosophila melanogaster. Environ Microbiol 2018;21:3259-68.

Unckless RL, Boelio LM, Herren JK et al. Wolbachia as populations within individual insects: causes and consequences of density variation in natural populations. Proc $R$ Soc B Biol Sci 2009;276:2805-11.

Valette V, Bitome Essono P-Y, Le Clec'h W et al. Multi-infections of feminizing Wolbachia strains in natural populations of the terrestrial isopod Armadillidium vulgare. PLoS One 2013;8:e82633.

van Opijnen T, Breeuwer JA. High temperatures eliminate Wolbachia, a cytoplasmic incompatibility inducing endosymbiont, from the two-spotted spider mite. Exp Appl Acarol 1999;23:871-81.

Veneti Z, Clark ME, Karr TL et al. Heads or tails: host-parasite interactions in the Drosophila-Wolbachia system. Appl Environ Microbiol 2004;70:5366-72. 
Vigneron A, Masson F, Vallier A et al. Insects recycle endosymbionts when the benefit is over. Curr Biol 2014;24:2267-73.

Voronin D, Cook DAN, Steven A et al. Autophagy regulates Wolbachia populations across diverse symbiotic associations. Proc Natl Acad Sci 2012;109:E1638-46.

Werren JH, Baldo L, Clark ME. Wolbachia: master manipulators of invertebrate biology. Nat Rev Microbiol 2008;6:741-51.

White PM, Pietri JE, Debec A et al. Mechanisms of horizontal cell-to-cell transfer of Wolbachia spp. in Drosophila melanogaster. Appl Environ Microbiol 2017;83:e03425.

White PM, Serbus LR, Debec A et al. Reliance of Wolbachia on high rates of host proteolysis revealed by a genome-wide RNAi screen of Drosophila cells. Genetics 2017b;205:1473-88.

Xi Z, Gavotte L, Xie Y et al. Genome-wide analysis of the interaction between the endosymbiotic bacterium Wolbachia and its Drosophila host. BMC Genomics 2008;9:1.

Yang K, Xie K, Zhu YX et al. Wolbachia dominate Spiroplasma in the co-infected spider mite Tetranychus truncatus. Insect Mol Biol 2019;29:19-37.

Zug R, Hammerstein P. Wolbachia and the insect immune system: what reactive oxygen species can tell us about the mechanisms of Wolbachia-host interactions. Front Microbiol 2015;6:1201. 


\section{FIGURES AND TABLES}

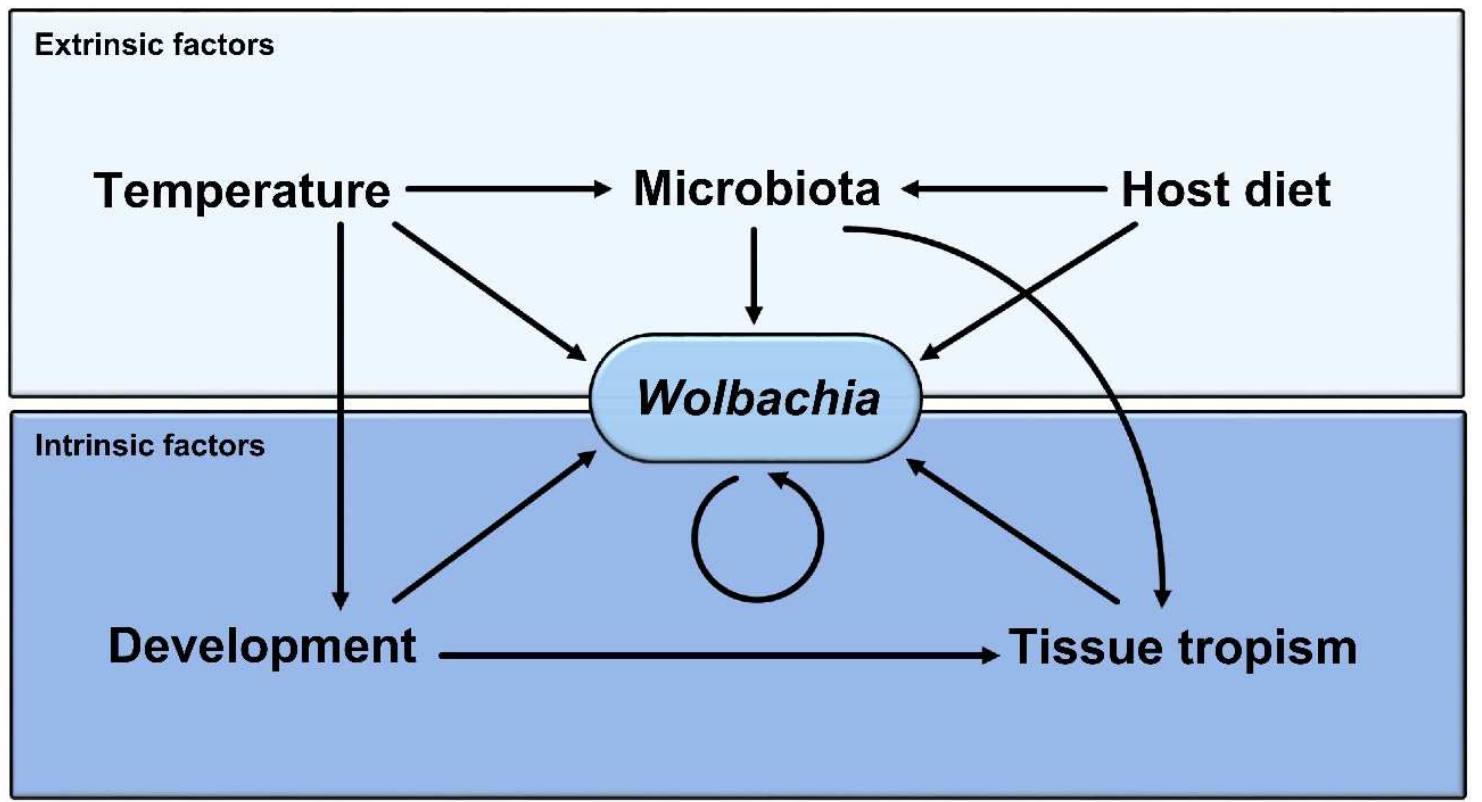

Figure 1. Factors shaping Wolbachia titers. Extrinsic (top panel) and intrinsic (bottom panel) factors and their potential interactions (arrows) are indicated. Additional interactions among extrinsic/intrinsic factors may occur (e.g. host diet/development, microbiota/development) but their impact on Wolbachia titer dynamics is yet unclear.

Table 1. Genetic basis of Wolbachia titer regulation. Loci shown to in vivo influence Wolbachia titers.

\begin{tabular}{|c|c|c|c|}
\hline Loci & Target & Model & Reference \\
\hline Metalloprotease $^{h}$ & Proliferation & A. aegypti/wMelPop-CLA & Hussain et al. 2011 \\
\hline MicroRNA aae-miR-2940 ${ }^{h}$ & Proliferation & A. aegypti/wMelPop-CLA & Hussain et al. 2011 \\
\hline Gurken $^{h}$ & Proliferation/Trafficking & D. melanogaster/w Mel & Serbus et al. 2011 \\
\hline MicroRNA aae-miR-12 ${ }^{h}$ & Proliferation/Maintenance & A. aegypti/wMelPop-CLA & Osei-Amo et al. 2012 \\
\hline Octomom $^{w}$ & Proliferation & D. melanogaster/wMelPop & Chrostek et al. 2013 \\
\hline Chickadee $^{h}$ & Trafficking & D. melanogaster/w Mel & Newton et al. 2015 \\
\hline Quail $^{h}$ & Trafficking & D. melanogaster $/ w \mathrm{Mel}$ & Newton et al. 2015 \\
\hline Wolbachia actin-localizing effector $1^{w}$ & Proliferation/Trafficking & D. melanogaster $/ w \mathrm{Mel}$ & Sheehan et al. 2016 \\
\hline Ubiquitin conjugating enzyme $6^{h}$ & Proliferation/Maintenance & D. melanogaster/w Mel & White et al. 2017b \\
\hline Quantitative-trait loci $1^{h}$ & Proliferation/Maintenance & C. quinquefasciatus/wPip & Emerson and Glaser, 2017 \\
\hline Quantitative-trait loci $2^{h}$ & Proliferation/Maintenance & C. quinquefasciatus/wPip & Emerson and Glaser, 2017 \\
\hline Wolbachia density suppressor $^{h}$ & Trafficking & N. vitripennis/wVitA & Funkhouser-Jones et al. 2018 \\
\hline Ribosomal protein L27A ${ }^{h}$ & Proliferation/Maintenance & D. melanogaster $/ w \mathrm{Mel}$ & Grobler et al. 2018 \\
\hline Ribosomal protein $\mathrm{S} 3^{h}$ & Proliferation/Maintenance & D. melanogaster $/ w \mathrm{Mel}$ & Grobler et al. 2018 \\
\hline
\end{tabular}

${ }^{h}$ Host encoded; ${ }^{w}$ Wolbachia encoded 